\title{
Lichen Planopilaris is a Common Scarring Alopecia among Iraqi Population*
}

\author{
Khalifa E. Sharquie $^{1 \#}$, Adil A. Noaimi ${ }^{2}$, Ammar F. Hameed ${ }^{2}$ \\ ${ }^{1}$ Scientific Council of Dermatology \& Venereology-Iraqi Board for Medical Specializations, Baghdad, Iraq; ${ }^{2}$ Department of Derma- \\ tology \& Venereology, College of Medicine, University of Baghdad, Baghdad, Iraq. \\ Email: "ksharquie@ymail.com, adilnoaimi@yahoo.com, amarfaisal1976@yahoo.com
}

Received May $18^{\text {th }}, 2013$; revised June 20 ${ }^{\text {th }}, 2013$; accepted June $30^{\text {th }}, 2013$

Copyright (c) 2013 Khalifa E. Sharquie et al. This is an open access article distributed under the Creative Commons Attribution License, which permits unrestricted use, distribution, and reproduction in any medium, provided the original work is properly cited.

\begin{abstract}
Background: Scarring alopecia like discoid lupus erythematosus, lichenplanopilaris and scarring folliculitis are common skin problems where differentiations between them are often difficult in many cases. Objective: To report, study and evaluate cases of lichen planopilaris and characterize the different clinical points in favor of this disease. Patients and Methods: This case series descriptive study was done at Department of Dermatology-Baghdad Teaching Hospital from January 2010 to November 2012. Sixty seven patients were included where histological and dermatological examinations were carried out to all patients regarding all relevant points related to the disease. Punch biopsies were performed from ten patients for histopathological study. Results: The mean age of onset of the disease was $36.7 \pm 5$ years with a mean duration of the disease being $4.4 \pm 3$ years. The male to female ratio was 1.4:1. The characteristic lesions were pigmented scarring moth eaten alopecia surrounded by pigmented hyperkeratotic follicular papules involving mainly the fronto-vertical and parietal scalp. Conclusions: Lichen planopilaris is a common cause of scalp scarring alopecia in adult and it has many characteristic clinical features which can facilitate differentiation from other pathologic scalp conditions like discoid lupus erythematosus.
\end{abstract}

Keywords: Cicatricial Alopecia; Lichen Planopilaris; Moth Eaten Alopecia

\section{Introduction}

Lichen planopilaris (LPP) is a chronic inflammatory disease characterized by lymphocytic destruction of the hair follicle resulting in progressive scalp scarring alopecia. The cause is unclear but autoimmune pathology seems to be the most accepted theory [1]. Women are affected more than men and clinical presentation includes grouped keratotic follicular papules surrounded by erythema. Early disease is usually confirmed by histological examination, however a late disease often has no more specific signs making it hard to separate from other scarring scalp conditions even with utilizing the histopathological examination [2].

The North American Hair Research Society (NAHRS) placed LPP in the lymphocytic group. Although, LPP is considered a rare disease but it is the most frequent cause of adult primary scarring alopecia. According to the NAHRS classification, LPP has been subdivided into 3

\footnotetext{
*Disclosure: This study was an independent study and not funded by any pharmaceutical company.

${ }^{\#}$ Corresponding author.
}

variants: classic LPP, frontal fibrosing alopecia (FFA), and Lassueur Graham-Little Piccardi syndrome [3].

Scarring alopecia is considered as one of the most disfiguring problems that cause great cosmetic concern for the patients. In our daily practice the most common causes of scaring alopecia are trauma (including burn), LPP, discoid lupus erythematosus (DLE), fungal infection (kerion) and striae distensae-like lesions scarring alopecia [4].

The aim of the present study was to study and evaluate the characteristic clinical features which can differentiate classic LPP from other scarring scalp diseases.

\section{Patients and Methods}

This case seris descriptive study involved a total of 67 patients diagnosed as LPP and registered at the Department of Dermatology and Venerology-Baghdad Teaching Hospital between January 2010 to November 2012.

Clinical data including demographics: gender, age of patient at onset of the disease, duration of the disease, symptoms and signs of LPP in the scalp and other body 
sites, site and extent of the disease. Medical photographic documentation of the lesions were done using Canon Digital IXUS 85IS camera. The extent of disease was adapted from Dr. Pric's visual aid for estimating percentage of scalp hair loss in which one can divide the scalp into 4 quadrants and estimate the percentage of the scalp surface that all the alopecic areas would occupy if placed together [5]. Histopathological examination was achieved in 10 patients. Six mm punch biopsies were taken from the scaly follicular border of the lesion to be submitted for vertical sectioning and Haematoxylin-Eosin staining. Formal consent was obtained from each patient after full explanation of the goals and the nature of the study to them and the study was approved by the ethical committee of Iraqi Board for Medical Specializations. Simple descriptive statistical analysis was carried out.

\section{Results}

Sixty seven patients with LPP were evaluated. Their ages ranged between 20 to 65 years with a mean age \pm SD of onset of the disease was $36.7 \pm 5$ years. The mean duration of their disease was $4.4 \pm 3$ years with a range of 0.5 - 15 years. The male to female ratio was 1.4:1 (39 men versus 28 women). Fifty five (79\%) patients were symptomatic with increased hair shedding observed in 45 (67\%) patients, pruritus in 40 (59.7\%) patients and scalp tenderness in 36 (53.7\%) patents.

Parietal and fronto-vertical scalp areas were the most commonly involved parts where it was affected in 50 (74\%) patients and in 41 (61\%) patients respectively (Table 1).

Twenty nine (43\%) patients having 51\% - 75\% of their scalp involved by pigmented patchy scarring hair loss which is surrounded at its periphery by acuminate pigmented hyperkeratotic follicular papules. This pattern of hair loss was irregular in all cases simulating the so called moth eaten alopecia seen in secondary syphilis and alopecia areata (Figure 1). Extra-cranial lichen planus was found in 18 (26.8\%) patients with the trunk being the most commonly affected part in 7 (10\%) patients.

The follicular truncal involvement was asymptomatic and often unnoticed by the patients (Figure 2). Other extra-cranial involvements are shown in Table $\mathbf{1 .}$

Histology of the examined patients were almost diagnostic of LPP through demonstration of lymphocytic lichenoid infiltration of the upper part of follicular epithelium with vacuolar changes in its basal layer, presence of colloid bodies and melanophages, the inflammatory process was found to affect the upper permanents segment of the hair follicle at the attachment of erector pilli muscle (bulge region) causing dermal fibrosis while the lower segment of the hair follicle including hair matrix was not affected by this pathologic process.
Table 1. Clinical characteristics of the LPP patients.

\begin{tabular}{lc}
\hline Clinical findings & No. (\%) \\
\hline Site of the LPP & $41(61)$ \\
Frontal scalp & $50(74)$ \\
Parietal scalp & $17(25)$ \\
Temporal scalp & $35(52)$ \\
Occipital scalp & \\
Symptoms of LPP & $45(67)$ \\
Hair shedding & $40(60)$ \\
Pruritus & $36(54)$ \\
Tenderness & \\
Extent of scalp involvement & $9(13.4)$ \\
1\% - 25\% & $22(33)$ \\
$26 \%$ - 50\% & $29(43)$ \\
$51 \%$ - 75\% & $7(10)$ \\
$76 \%$ - 100\% & $19(28)$ \\
Extra-cranial LP & $11(16)$ \\
Trunk & $7(10)$ \\
Mucous membrane & $1(1.5)$ \\
Legs & $1(1.5)$ \\
Face & $1(1.5)$ \\
Nails & $1(1.5)$ \\
Eyebrows & $1(1.5)$ \\
Axilla and groin &
\end{tabular}

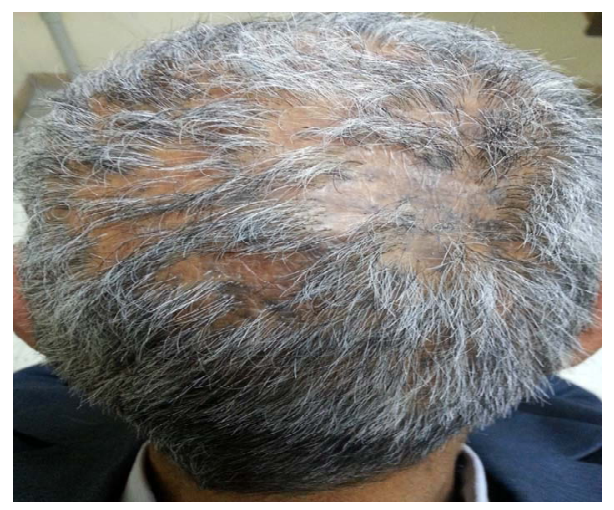

Figure 1. Pigmented scarring moth eaten alopecia involving the occipital and parietal scalp of an elderly man with lichen planopilaris.

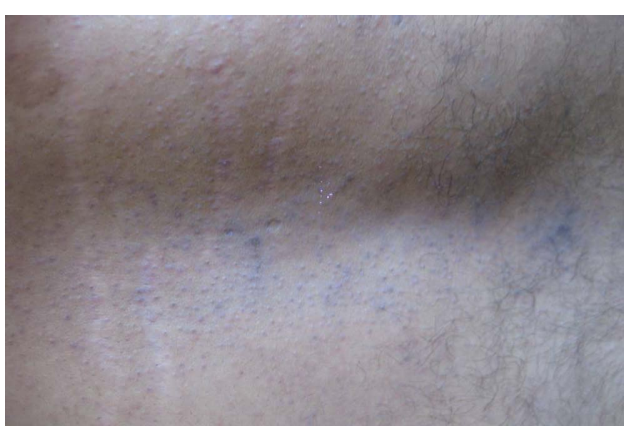

Figure 2. Pigmented lichenoidfollicular papules distributed in the anterior trunk of male patient with lichen planopilaris. 


\section{Discussion}

Scarring alopecia represents a challenge for both patient and the treating dermatologist where early diagnosis is essential to provide a guide for the initiation of an early and effective therapy to save the follicular apparatus from the irreversible damage caused by a variety of diseases. From the daily clinical practice, we think that LPP is the commonest cause of adult scarring alopecia in the present time. Many Iraqi dermatologists are unaware about this clinical observation and they often misdiagnosed LPP as DLE. The main objective of the present study is to characterize the main clinical features of LPP that can differentiate it from other similar scarring conditions like DLE.

The mean age of onset inpatients of the present work was $36.7 \pm 5$ years which is considered younger than that reported by Tan et al. [5] where the mean age was 47.4 years and that of Cevasco et al. with a mean age was 48.9 years [6].

The present study showed that males were more affected than males with a ratio of 1.4:1.These differences in age and sex distribution can be attributed to the racial differences as the present study was mainly among Arab while previous studies were mainly among Caucasian $[7,8]$.

Parietal and fronto-vertical regions were the most frequently involved part of the scalp in the present study which is comparable to what had been reported in the medical literatures $[6,9,10]$.

Scarring alopecia was severe in $43 \%$ of patient as $51 \%$ - $75 \%$ of their scalp surface area was affected by the scarring process. This can be explained partly by the delayed or misdiagnosis with other similar conditions.

All patients had prominent and characteristic multifocal irregular areas of patchy scarring hair loss resembling moth eaten alopecia which is similar to what has been seen in secondary syphilis and alopecia areata [11,12] ( Figures 3 and 4).

However, loss of follicular orifices can readily discriminate the scarring moth eaten alopecia of LPP from that of non scarring moth eaten alopecia in secondary syphilis or alopecia areata [12]. This observation was not well documented in the medical literature.

Scarring alopecia of the scalp can be associated with other regional involvements as the present work showed that $26.8 \%$ of patients had extracranial involvement which was in agreement with many published reports [7, 8]. Most patients were unaware about the presence of these small asymptomatic truncal follicular papules unless it closely looked for by the examining dermatologist (Figure 2). Hence careful examinations of all body regions are mandatory in evaluation of scarring scalp conditions.

All patients had prominent and characteristic multi-

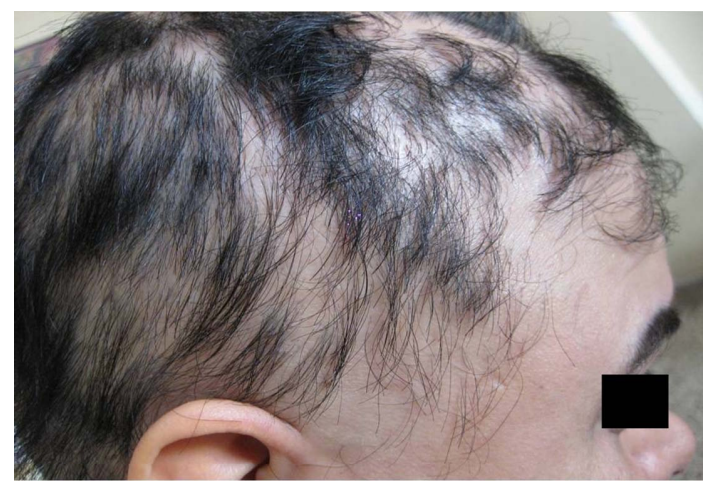

Figure 3. The non-scarringmoth eaten alopecia in male patient with alopecia areata.

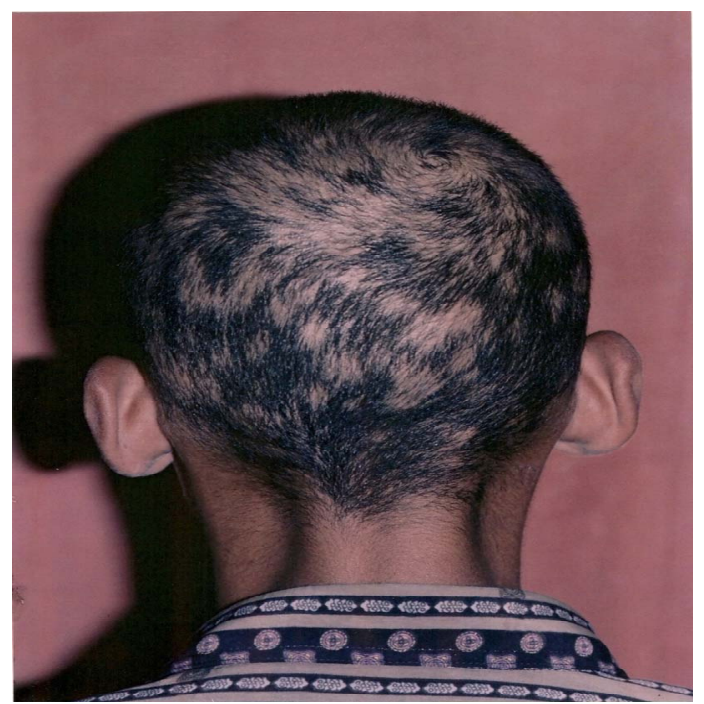

Figure 4. Typical moth eaten alopecia of secondary syphilis in male patient.

focal irregular areas of patchy scarring hair loss resembling moth eaten alopecia which is similar to what has been seen in secondary syphilis and alopecia areata [12, 13] (Figures 3 and 4).

However loss of follicular orifices can readily discriminate the scarring moth eaten alopecia of LPP from that of non scarring moth eaten alopecia of secondary syphilis or alopecia areata [13].

The main diagnostic challenge for LPP is DLE. However there are many clinical and histopathological points which can help the dermatologists to differentiate between these two primary lymphocytic cicatricial conditions as follows:

- The early lesions of LPP are pigmented follicular papules while the early presentation of scalp DLE is single or multiple scaly erythematous patches (Figure 5).

- The scarring process in LPP is early and pigmented while scarring in DLE is usually late and erythematous 


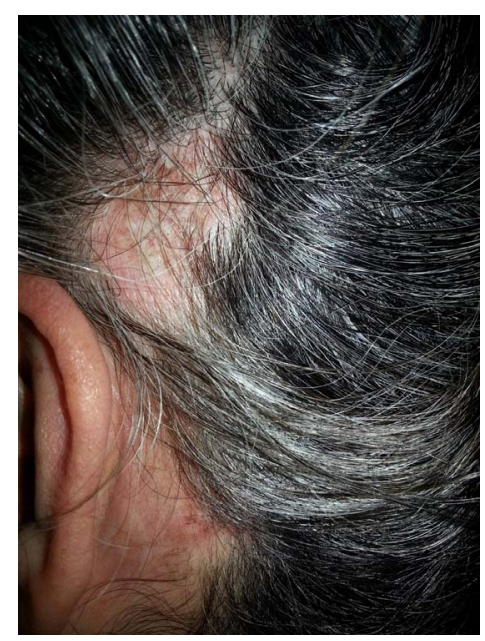

Figure 5. Erythematous scarring alopecia in female patient with discoid lupus erythematosus.

- Irregular scarring areas that simulate moth eaten alopecia are characteristic feature of LPP scarring and can be considered as the most important differentiating point between DLE and LPP.

- The activity of the disease is mainly central in DLE lesions while the activity of LPP is mainly at the periphery of the scarring patch in the form of hyperkeratotic follicular papules [6].

- The associated manifestation of other body regions also can help approach the diagnosis; The lesions in DLE commonly affect the sun exposed parts, particularly on the cheeks, nose and the ears $[13,14]$ with typical discoid lesions, while LPP lesions, as demonstrated by the present work, commonly present with a symptomatic truncal follicular papules which might be scarring or not (Figure 2).

- Although both conditions share lichenod lymphocytic infiltration of basal epithelium and follicular structure with vacuolar interface dermatitis, but DLE lesions are characterized by deeper follicular inflammation and perieccrine lymphocytic infiltration while the perifollicular inflammation in LPP is more superficial and limited to the upper segment of the hair follicle [8] (Figure 6).

- The pathologic changes in active LPP are mainly follicular without affection of the epidermis while in DLE the changes are both epidermal (vacuolar interface dermatitis) and dermal (interfollicular mucin deposition) [15]. We also noticed an important observation where the inflammatory process involved the area of bulge region causing fibrosis and leaving the lower segment of the hair follicle intact. This pathologic finding may be responsible for the permanent damage to the hair organ and support the theory that the bulge region is the site of hair regeneration [16].

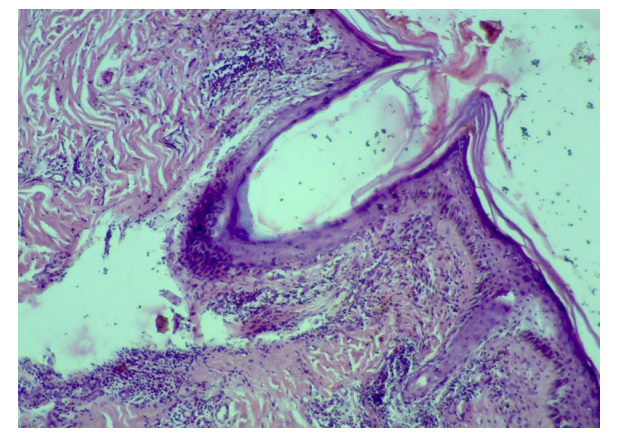

Figure 6. Lichen planopilaris showing follicular hyperkeratosis and superficial perifollicularlichenoid lymphocytic infiltrations causing dermal fibrosis. (H\&E stain $\times 40$ ).

- Thickening of basement membrane and mucin deposition in the dermis is a feature of DLE [17].

- Other laboratory abnormalities like antinuclear factor are frequently positive in DLE but not in LPP [14]. Accordingly DLE represents a systemic disease with follicular involvement while LPP can be considered as localized skin problem.

- Early treatment of DLE can induce rapid hair regrowth with little or no scarring while in LPP, the scarring is aggressive from the start and the aim of the medical intervention is to stop the early progress of the disease. Hence very early diagnosis is mandatory.

In conclusion, scarring alopecia is a common problem among adult population which can end by Pseudopelade of Brocq. Although LPP and DLE can be so similar both clinically and histologically, still a careful clinical examination remains the gold standard in establishing the final diagnosis. Scarring moth eaten alopecia is the main clinical differentiating point from other scarring scalpconditions like DLE.

\section{REFERENCES}

[1] D. N. Silvers, B. E. Katz and A. W. Young, "Pseudopelade of Brocq Is Lichen Planopilaris: Report of Four Cases That Support This Nosology," Cutis, Vol. 51, No. 2, 1993, pp. 99-105.

[2] H. Kang, A. A. Alzolibani, N. Otberg and J. Shapiro, "Lichen Planopilaris,” Dermatology and Therapy, Vol. 21, No. 4, 2008, pp. 249-256. doi:10.1111/j.1529-8019.2008.00206.x

[3] E. A. Olsen, W. F. Bergfeld, G. Cotsarelis, V. H. Price, J. Shapiro, R. Sinclair, A. Solomon, L. Sperling, K. Stenn, D. A. Whiting, O. Bernardo, M. Bettencourt, C. Bolduc, V. Callendar, D. Elston, J. Hickman, M. Ioffreda, L. King, C. Linzon, A. McMichael, J. Miller, F. Mulinari and R. Trancik, "Summary of North American Hair Research Society (NAHRS)-Sponsored Workshop on Cicatricial Alopecia, Duke University Medical Center, February 10 and 11, 2001," Journal of the American Academy of Dermatology, Vol. 48, 2003, pp. 103-110. doi:10.1067/mjd.2003.68 
[4] K. E. Sharquie, M. M. Al-Waiz and A. A. Al-Nuaimy, "Striae Distensae-Like Lesions. A Cause of Scarring Alopecia among Children,” Saudi Medical Journal, Vol. 23, No. 12, 2002, pp. 1489-1491.

[5] E. L. Khoury, V. H. Price, M. M. Abdel-Salam, M. Stern and J. S. Greenspan, "Topical Minoxidil in Alopecia Areata: No Effect on the Perifollicular Lymphoid Infiltration," Journal of Investigative Dermatology, Vol. 99, 1992, pp. 40-47. doi:10.1111/1523-1747.ep12611409

[6] E. Tan, M. Martinka, N. Ball and J. Shapiro, "Primary Cicatricialalopecias: Clinicopathology of 112 Cases,” Journal of the American Academy of Dermatology, Vol. 50, No. 1, 2004, pp. 25-32. doi:10.1016/j.jaad.2003.04.001

[7] N. Cevasco, W. Bergfeld, B. Remzi and H. Ramirez de Knott, "A Case-Series of 29 Patients with Lichen Planopilaris: The Cleveland Clinic Foundation Experience on Evaluation, Diagnosis, and Treatment," Journal of the American Academy of Dermatology, Vol. 57, No. 1, 2007, pp. 47-53. doi:10.1016/j.jaad.2007.01.011

[8] D. A. Mehregan, H. M. Van Hale and S. A. Muller, "Lichen Planopilaris: Clinical and Pathologic Study of FortyFive Patients," Journal of the American Academy of Dermatology, Vol. 27, No. 6, 1992, pp. 35-42. doi:10.1016/0190-9622(92)70290-V

[9] W. F. Bergfeld and D. M. Elston, "Cicatricial Alopecia," In: E. A. Olsen, Ed., Disorders of Hair Growth: Diagnosis and Treatment," McGraw-Hill, New York, 2003, pp. 363398.

[10] K. Wiedemeyer, W. B. Schill and C. Löser, "Diseases on Hair Follicles Leading to Hair Loss Part II: Scarring Alopecias,” Skinmed, Vol. 3, No. 5, 2004, pp. 266-269.
[11] M. Y. Bi, P. R. Cohen, F. W. Robinson and J. M. Gray, "Alopecia Syphilitica-Report of a Patient with Secondary Syphilis Presenting as Moth-Eaten Alopecia and a Review of Its Common Mimickers,” Dermatology Online Journal, Vol. 15, No. 10, 2009, p. 6.

[12] J. Y. Lee and M. L. Hsu, “Alopeciasyphilitica, a Simulator of Alopeciaareata: Histopathology and Differential Diagnosis,” Journal of Cutaneous Pathology, Vol. 18, No. 2, 1991, pp. 87-92. doi:10.1111/j.1600-0560.1991.tb00133.x

[13] S. K. Clark, "Cutaneous Lupus Erythematosus, Recognition of Its Many Forms,” Postgraduate Medicine, Vol. 79, No. 5, 1986, pp. 195-203.

[14] M. K. Al-Hattab and M. Al-Waiz, "Discoid Lupus Erythematosus in Iraqi Patients: A Clinical and Histopathological Study," Annals of Saudi Medicine, Vol. 24, No. 4, 2004, pp. 289-292.

[15] D. Whiting, "Cicatricial Alopecia: Clinico-Pathological Findings and Treatment," Clinics in Dermatology, Vol. 19, No. 2, 2001, pp. 211-225. doi:10.1016/S0738-081X(00)00132-2

[16] N. Mobini, S. Tam and H. Kamino, "Possible Role of the Bulgeregion in the Pathogenesis of Inflammatory Scarring Alopecia: Lichenplanopilaris as the Prototype,” Journal of Cutaneous Pathology, Vol. 32, No. 10, 2005, pp. 675-679. doi:10.1111/j.0303-6987.2005.00399.x

[17] L. Sperling, "Scarring Alopecia and the Dermatopathologist,” Journal of Cutaneous Pathology, Vol. 28, No. 7, 2001, pp. 333-342. doi:10.1034/j.1600-0560.2001.280701.x 